\title{
shRNA-mediated NP knockdown inhibits the apoptosis of cardiomyocytes induced by H1N1pdm2009 influenza virus
}

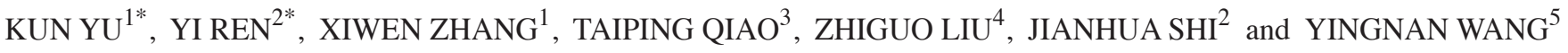 \\ Departments of ${ }^{1}$ Cardiology and ${ }^{2}$ Breast and Thyroid, Huai'An First People's Hospital, Nanjing Medical University, \\ Huai'an, Jiangsu 223300; Departments of ${ }^{3}$ Surgery, ${ }^{4}$ Medical Oncology and ${ }^{5}$ Cardiovascular Medicine, \\ Wulanchabu Central Hospital, Jining, Wulanchabu 012000, P.R. China
}

Received April 2, 2016; Accepted February 1, 2017

DOI: $10.3892 / \mathrm{mmr} .2017 .6728$

\begin{abstract}
Acute influenza-associated myocarditis varies in clinical severity ranging between asymptomatic and fulminant varieties. The most severe cases can result in impaired cardiac function-associated mortality; however, the mechanism underlying the development of viral myocarditis has yet to be fully elucidated. The present study investigated the apoptosis induced in H9C2 cardiomyocytes by infection with the H1N1pdm2009 virus. The H9C2 cells were transfected with nucleoprotein (NP)-specific short hairpin (sh) RNA, and viral replication was re-evaluated in H9C2 cells infected with the H1N1pdm2009 virus, as was the apoptosis induced by the virus. Reverse transcription-quantitative polymerase chain reaction and western blot analysis were performed to measure the expression of NP and apoptosis-associated molecules. A plaque forming assay was used to quantify viral replication in H9C2 cells. An MTT assay and flow cytometric analysis were performed to determine the virus-associated alterations in cellular viability and apoptosis, respectively. Results demonstrated that the H1N1pdm2009 virus replicated effectively in H9C2 cells and promoted apoptosis in association with the viral infection. The expression levels of apoptosis-associated markers, including released cytochrome $c$ and activated caspase-3 were markedly promoted in the H1N1pdm2009-infected H9C2 cells. However, the NP-specific shRNA-mediated NP knockdown significantly inhibited viral infection in the cells. The virus-induced apoptosis of the H9C2 cells was also significantly reduced by the shRNA, which occurred via a decrease in the number of apoptotic cells through downregulating the levels of apoptosis-associated markers. Taken together, the
\end{abstract}

Correspondence to: Dr Yingnan Wang, Department of Cardiovascular Medicine, Wulanchabu Central Hospital, 157 Jiefang Road, Jining, Wulanchabu 012000, P.R. China

E-mail: yingnanwangwlcb@163.com

*Contributed equally

Key words: short hairpin RNA, nucleoprotein knockdown, apoptosis, cardiomyocytes, influenza virus present study demonstrated the key pathogenic role of NP in H1N1pdm2009-induced apoptosis of cardiomyocytes, and this marker of the influenza virus may be important in influenza virus-associated acute myocarditis. In addition, NP-specific shRNA may be an effective agent for inhibiting influenza virus-induced apoptosis in cardiomyocytes or in influenza virus-associated acute myocarditis.

\section{Introduction}

The influenza A/California/07/2009 (H1N1) virus outbreak in Mexico and the United States in 2009 was found to be genetically correlated with swine influenza viruses (1). The H1N1 virus subsequently spread to other countries and caused severe outbreaks around the world. Up to $10 \%$ of severe influenza infections cause pneumonia with myocarditis or acute myocardial infarction (2-4). Individuals with underlying chronic cardiovascular diseases are at higher risk of influenza A virus (IAV)-induced myocarditis (5). However, the mechanism underlying the viral myocarditis remains to be fully elucidated.

The whole genome of IAV contains eight negative-sense RNA segments, comprising PB1, PB2, PA, HA, nucleoprotein (NP), neuraminidase (NA), (M1 and NS1), which encode $>10$ proteins. The IAV genome is variable for the low fidelity of RNA polymerase and recombination between strains (6), allowing viruses to evade host immunity and outbreak cyclically. Influenza virus infection leads to the activation of various intracellular signaling pathways, which not only partially increase the antiviral response, but also support viral replication simultaneously $(7,8)$. Several IAV proteins have been implicated in apoptosis in infected cells. Previous studies have indicated the NS1 protein of IAV downregulates apoptosis in early infection to support virus replication (9). NA can activate transforming growth factor (TGF)-h, a known inducer of apoptosis $(10,11)$. PB1 and PB2 are also considered to be involved in the induction of apoptosis in infected cells (12). NP is phosphorylated and cleaved by proteases in infected cells, and IAVNP (56 kDa; NP56) is converted proteolytically into a 53-kDa form (NP53) (13), NP cleavage appears to prevent packaging of viral ribonucleoprotein into virus particles, with only uncleaved NP56 found assembled into virions (14). To date, the association between the level of NP and apoptosis remains to be fully elucidated. 
In the present study, the replication of IAV in H9C2 cells was suppressed using short hairpin (sh) RNA against NP, and the apoptosis induced by virus infection was examined. The results aimed to determine whether viral replication is associated with the level of apoptosis induced.

\section{Materials and methods}

Cell culture and production of the shRNA-NP lentivirus. Cardiomyoblast H9C2 cells (American Type Culture Collection; ATCC; Manassas, VA, USA) and 293T cells were cultured in Dulbecco's modified Eagle's medium (DMEM; Gibco; Thermo Fisher Scientific, Inc., Waltham, MA, USA), supplemented with $10 \%$ fetal calf serum (FCS; Gibco; Thermo Fisher Scientific, Inc.), $100 \mathrm{U} / \mathrm{ml}$ penicillin/streptomycin and $2 \mathrm{mM}$ L-glutamine (growth medium), and were maintained at $37^{\circ} \mathrm{C}$ in a humidified $5 \% \mathrm{CO}_{2}$ atmosphere. For the production of the shRNA-NP lentivirus, cells were seeded in a T25 tissue culture plate at a density of $2 \times 10^{5}$ cells $/ \mathrm{ml}$, when $50-70 \%$ confluent, and were transfected for $6 \mathrm{~h}$ with an shRNA-NP plasmid and a control-shRNA plasmid, using Lipofectamine 2000 as the transfection reagent (Invitrogen; Thermo Fisher Scientific, Inc.) at $37^{\circ} \mathrm{C}$ in a humidified $5 \% \mathrm{CO}_{2}$ atmosphere. After $48 \mathrm{~h}$, the supernatant was harvested, filtered using a $0.2 \mu \mathrm{m}$ filter and stored at $-80^{\circ} \mathrm{C}$.

IAV virus propagation. The influenza A/H1N1pdm2009 virus (CA07) was propagated in Madin Darby canine kidney cells (line CV-1; ATCC). The cells were passaged in DMEM containing $10 \%$ bovine FCS. For infection of the H9C2 cells, 2-day-old confluent cell monolayers were incubated with the virus at the indicated multiplicity of infection (MOI) for $1 \mathrm{~h}$ at $37^{\circ} \mathrm{C}$. Following infection, the cells $\left(2 \times 10^{4}\right.$ cells/well $)$ were washed and incubated with DMEM at $37^{\circ} \mathrm{C}$ for different durations $(0,8,12,24$ and $48 \mathrm{~h})$ and were then prepared for further examination. The titers of infectious virus in the supernatants were determined using common plaque assays, as described previously $(15,16)$.

Evaluation of NP mRNA levels using reverse transcriptionquantitative polymerase chain reaction ( $R T-q P C R)$ analysis. The mRNA level of NP was determined using RT-qPCR analysis, as described previously (2). Briefly, total RNA was isolated from cells $\left(1 \times 10^{5}\right.$ cells/sample) using TRIzol ${ }^{\circledR}$ reagent (Invitrogen; Thermo Fisher Scientific, Inc.), according to the manufacturer's protocol. The cDNA was then synthesized by RT using a Reverse Transcription kit (Thermo Fisher Scientific, Inc.) with the NP-specific primer (5'-caccaaacgatcatatgaac-3'). qPCR was performed using the following primers $(10 \mathrm{nM})$ : NP, forward 5'-ccagaatgtget ctctaatg-3', reverse 5'-tcctttcaccgcagcacctg-3', as previously described (2). The quantification cycle (Cq) values (17) of the target gene were normalized to $\beta$-actin (forward 5'-gtaccetgg cattgccgaca-3', reverse 5'-ggactcgtcatactcctgcttgct-3') from the same sample as relative mRNA levels. Each sample was assessed in triplicate.

Western blot analysis. The H9C2 cells were collected and lysed with lysis buffer (Thermo Scientific, Rockford, IL, USA) on ice for $20 \mathrm{~min}$. The cell lysates were centrifuged at
$15,000 \mathrm{x} \mathrm{g}$ at $4^{\circ} \mathrm{C}$ for $30 \mathrm{~min}$. The supernatant was collected as the total cellular protein extract. Protein concentration was determined using the BCA Protein Assay kit (Kangwei Shiji Biotechnology Co., Ltd., Beijing, China). The samples of total cellular protein $(5 \mu \mathrm{g})$ were loaded onto a $10 \%$ SDS-PAGE. The separated proteins were electrophoretically transferred onto polyvinylidene difluoride membranes (Bio-Rad Laboratories, Inc., Hercules, CA, USA). The membranes were blocked overnight in blocking buffer containing PBS-T and $5 \%$ non-fat milk. The membranes were then incubated with primary antibodies against NP (1:800; cat. no. 11675-V08B-50; Sino Biological, Inc., Beijing, China), caspase-3 (1:500; cat. no. 3CSP03), cytochrome $c$ (CytC; 1:500; cat. no. sc-7159), $\mathrm{B}$ cell lymphoma-2-associated $\mathrm{X}$ protein (Bax; 1:500; cat. no. sc-493) and $\beta$-actin (1:500; cat. no. sc-81178), obtained from Santa Cruz Biotechnology, Inc. (Dallas, TX, USA), for $1 \mathrm{~h}$ at $37^{\circ} \mathrm{C}$, and then washed with PBS-T four times. Following incubation with the secondary horseradish peroxidase-conjugated antibody (1:400; cat. no. Ab131366; Abcam, Cambridge, UK) for $1 \mathrm{~h}$ at room temperature, the membranes were washed four times, treated with enhanced chemiluminescence reagent and exposed to X-ray film. Protein bands were semi-quantified using ImageJ software version $1.43 \mathrm{~b}$ (National Institutes of Health, Bethesda, MD, USA).

Determination of apoptosis. The percentages of apoptotic cells were determinedusing an Annexin V-FITCApoptosis Detection kit (EMD Millipore, Billerica, MA, USA). Briefly, the cells were incubated for $15 \mathrm{~min}$ in the dark with Annexin V-FITC and PI, according to the manufacturer's protocol. A total of $1.0 \times 10^{6}$ cells were washed twice with ice-cold PBS and incubated for $10 \mathrm{~min}$ in binding buffer, containing $5 \mu \mathrm{PI}$ and $5 \mu \mathrm{l}$ Annexin V-FITC. To quantify the apoptotic rate of cells in each group, at least 100,000 cells from each treatment group were examined using flow-cytometry, and the percentage of Annexin V-positive cells or Annexin V-plus-PI positive cells were calculated. All experiments were performed in triplicate. The activity of caspase-3 was analyzed using a caspase-3 activity assay kit (Cell Signaling Technology, Inc., Danvers, MA, USA).

MTT cell viability assay. The viability of the H9C2 cells was measured using the MTT method in 96-well plates. Briefly, following infection with the A/H1N1pdm2009 virus (multiplicity of infection=1) for 12,24 or $48 \mathrm{~h}, \mathrm{H} 9 \mathrm{C} 2$ cells $\left(1 \times 10^{4}\right.$ cells/well) at $80 \%$ confluence, were treated with $20 \mu 1$ of MTT solution (at a final concentration of $5 \mathrm{mg} / \mathrm{ml}$ ) for $4 \mathrm{~h}$ at $37^{\circ} \mathrm{C}$. The cell supernatant was carefully removed, and $200 \mu \mathrm{l}$ of DMSO was added to each well and mixed. The plate was placed in a $37^{\circ} \mathrm{C}$ incubator to dissolve air bubbles and the optical density (OD) 50 value of each well was measured at a $570 \mathrm{~nm}$ wavelength using a microplate reader (Thermo Fisher Scientific, Inc.). The results were calculated as follows: (A570 control wells-A570 treated wells)/(A570 control wells-A570 blank wells) x100\%.

Statistical analysis. Data are presented as the mean \pm standard deviation calculated from three independent experiments. For comparison between two groups, Student's t-test was used. For multiple comparisons among three or more groups, 

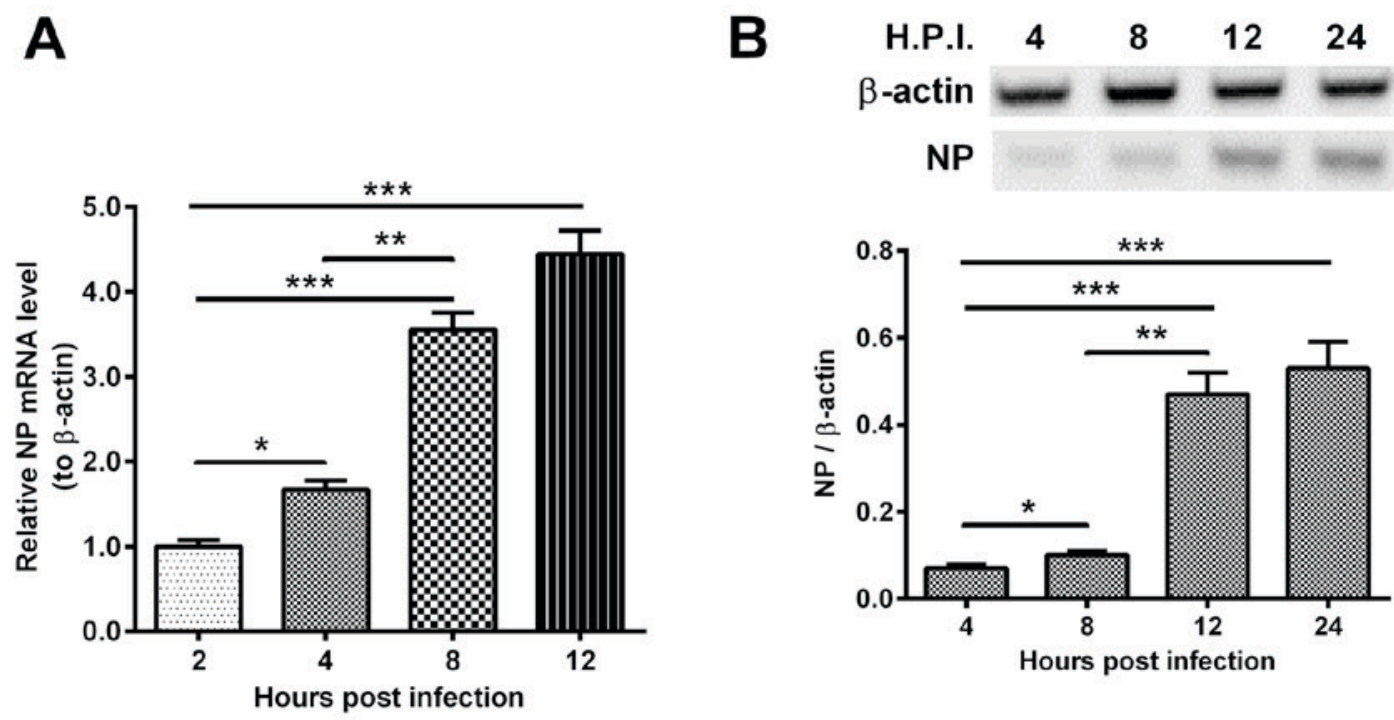

\section{C}
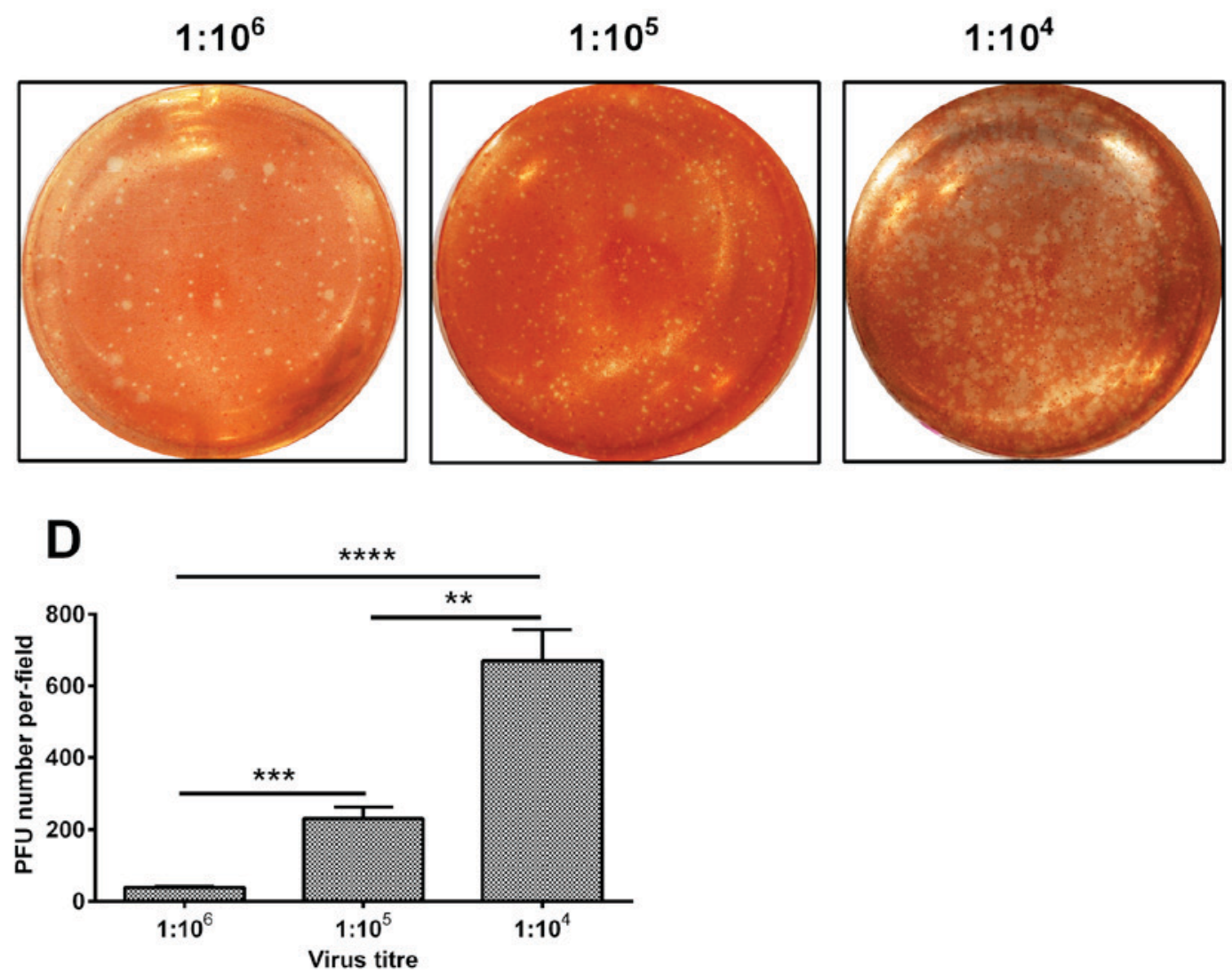

Figure 1. H1N1pdm2009 virus infects H9C2 cardiomyocytes. (A) Relative mRNA levels of influenza NP in H9C2 cardiomyocytes infected with 1 MOI H1N1pdm2009 virus for 2, 4, 8 or 12 h. (B) Western blot analysis of NP in the H1N1pdm2009 virus-infected (1 MOI) H9C2 cells. (C) Plaque-forming and (D) plaque counting of the H1N1pdm2009 virus in $\mathrm{H} 9 \mathrm{C} 2$ cells at a dilution of 1:104, 1:10 or 1:106. Photomicrographs were captured under $\mathrm{x} 1$ magnification. Quantitative data are the average of three independent results. ${ }^{*} \mathrm{P}<0.05,{ }^{* *} \mathrm{P}<0.01,{ }^{* * *} \mathrm{P}<0.001$ and ${ }^{* * * * *} \mathrm{P}<0.0001$. NP, nucleoprotein; MOI, multiplicity of infection; H.P.I, h post-infection.

one-way analysis of variance was used followed by a post hoc Newman-Keuls test. Analysis was performed using the SPSS software version 16.0 (SPSS, Inc., Chicago, IL, USA). $\mathrm{P}<0.05$ was considered to indicate a statistically significant difference.

\section{Results}

H1N1pdm2009 infection induces the apoptosis of cardiomyocytes. The $\mathrm{H} 9 \mathrm{C} 2$ cells were infected with the $1 \mathrm{MOI}$ H1N1pdm2009 strain. The relative mRNA and protein 
A

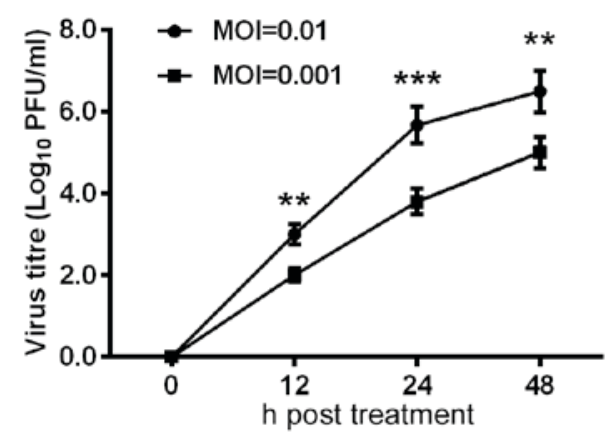

C

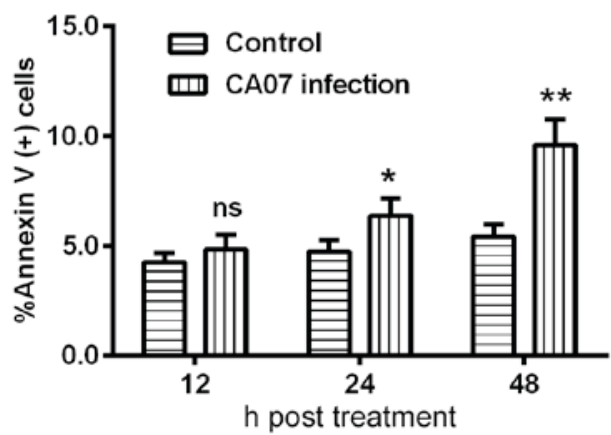

B

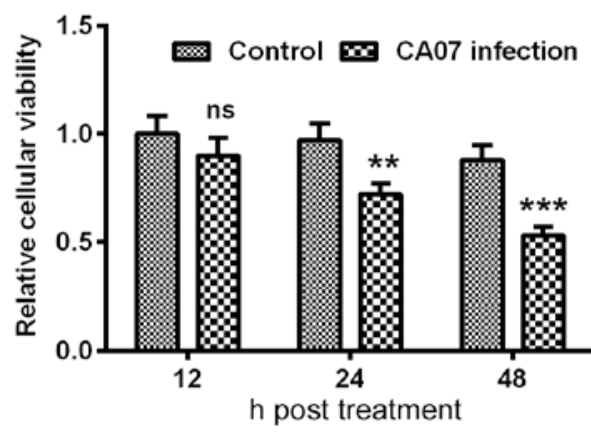

D

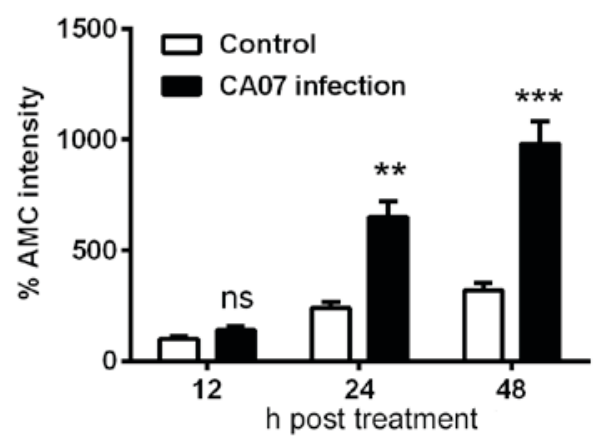

Figure 2. Apoptosis is induced by proliferation of the H1N1pdm2009 virus in H9C2 cells. (A) Growth curve of H1N1pdm2009 virus in H9C2 cells (MOI of 0.01 and 0.001). Vital titer was quantified at 12, 24 and $48 \mathrm{~h}$ using a plaque-forming assay. Results of MTT assays for (B) cellular viability, (C) induction of apoptosis and (D) activity of caspase-3 in the H9C2 cells infected with 1 MOI H1N1pdm2009 virus for 12, 24 and 48 h, respectively. Quantitative data are presented as the average of independent experiments performed in triplicate. ${ }^{*} \mathrm{P}<0.05,{ }^{* *} \mathrm{P}<0.01$ and ${ }^{* * *} \mathrm{P}<0.001$ vs. control. ns, no significance; $\mathrm{NP}$, nucleoprotein; MOI, multiplicity of infection.

levels of NP to $\beta$-actin were measured at $2,4,8$ and $12 \mathrm{~h}$ post-infection. As shown in Fig. 1A, the expression level of NP increased 4-fold at $12 \mathrm{~h}$ post-infection, compared with the level at $4 \mathrm{~h}$ post-infection $(\mathrm{P}<0.001)$. In addition, the level of NP increased 2 -fold at $8 \mathrm{~h}$ post-infection, compared with that at $2 \mathrm{~h}$ post-infection $(\mathrm{P}<0.01)$. Similar results were observed in the expression level of NP (Fig. 1B). The plaque forming ability of H1N1pdm2009 in the H9C2 cells was also assessed at dilutions of $1: 10^{4}, 1: 10^{5}$ and $1: 10^{6}$, respectively. As shown in Fig. 1C and D, the PFU number per-field at the $10^{4}$ dilution was the highest among the groups with statistical significance.

Subsequently, the growth curve of the H1N1pdm2009 virus in H9C2 cells was examined using an MOI of 0.01 and 0.001 , and the viral titer at each time point was determined using a plaque-forming assay. As shown in Fig. 2A, the group with an MOI of 0.01 presented with a higher titer, compared with that at the MOI of 0.001 at each time point. In addition, the relative cellular viabilities at each time point were assessed using an MTT assay; it was found that the cell viabilities were decreased by 30 and $50 \%$ at 24 and $48 \mathrm{~h}$ post-infection, respectively, and these differences were statistically significant (Fig. 2B).

Regarding the apoptosis induced by H1N1 infection, the present study quantified the H1N1 infection-induced apoptosis using an Annexin V kit, and observed a time-dependent increase in the percentage of Annexin $\mathrm{V}(+)$ cells and AMC intensity (Fig. 2C and D), indicating that H1N1 infection induced the apoptosis of $\mathrm{H} 9 \mathrm{C} 2$ cells.

shRNA efficiently inhibits the replication of H1N1pdm2009. The cells infected with 1 MOI of the H1N1pdm2009 virus were then infected by 1 MOI of Lenti-shRNA-NP-1, Lenti-shRNA-NP-2 or Lenti-shRNA-Con virus, and the relative mRNA levels of NP were measured at $12 \mathrm{~h}$ post-infection. The relative mRNA levels of NP in the shRNA-NP-treated groups were decreased by 60 and $65 \%$, compared with that in the shRNA control group, respectively (Fig. 3A). The results of the western blot analysis results also confirmed that shRNA transfection inhibited the expression of NP by 75 and $80 \%$, respectively (Fig. 3B and $\mathrm{C}$ ).

Subsequently, the H9C2 cells were infected with an MOI of 0.01 or 0.001 viral titer following the infection with $1 \mathrm{MOI}$ Lenti-shRNA-NP-1, Lenti-shRNA-NP-2 or Lenti-shRNA-Con virus. A growth curve was mapped at each time point, which was quantified using the plaque-forming assay. As shown in Fig. 4. A, no statistically significant differences were found among the groups at $12 \mathrm{~h}$ post-infection, however, the viral titers of the Lenti-shRNA-NP-1 and Lenti-shRNA-NP-2 groups were downregulated by $>40 \%$, compared with that of the Lenti-shRNA-con group $(\mathrm{P}<0.01)$. In addition, the relative 


\section{A}

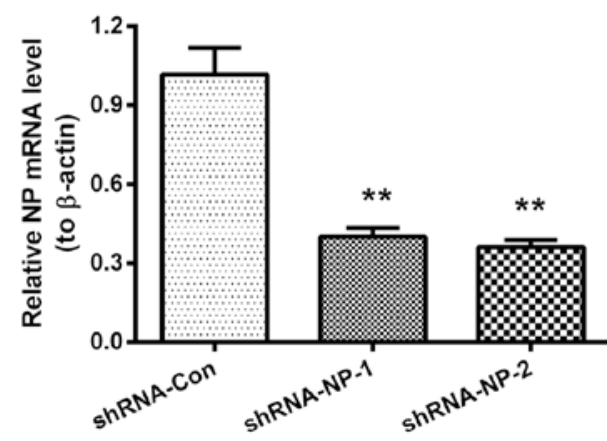

B

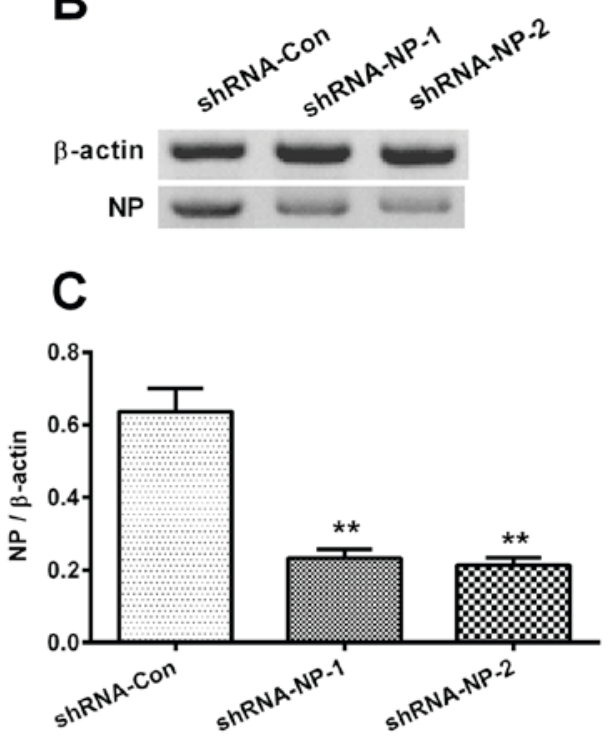

Figure 3. shRNA-mediated knockdown of NP in H1N1pdm2009 virus-infected $\mathrm{H} 9 \mathrm{C} 2$ cells. (A) Relative mRNA level and (B) results of western blot analysis of NP in H1N1pdm2009 virus-infected (1 MOI) H9C2 cells, which were also infected (1 MOI) with the Lenti-shRNA-NP-1, Lenti-shRNA-NP-2 or Lenti-shRNA-Con virus for $12 \mathrm{~h}$. (C) NP is presented relative to $\beta$-actin. Quantitative results are of independent experiments performed in triplicate. ${ }^{*} \mathrm{P}<0.05,{ }^{* *} \mathrm{P}<0.01$ and ${ }^{* * *} \mathrm{P}<0.001$ vs. shRNA-Con. ns, no significance; NP, nucleoprotein; MOI, multiplicity of infection; shRNA, short hairpin RNA; Con, control.

cellular viabilities of the Lenti-shRNA-NP-treated groups were significantly improved, compared with that in the control group at 24 or $48 \mathrm{~h}$ post-infection (Fig. 4B).

shRNA inhibits H1N1pdm2009-induced apoptosis. In order to examine the apoptosis inhibited by shRNA, the apoptotic rate of shRNA-treated cells were determined using an Annexin V kit, which revealed that apoptosis was significantly inhibited by $>20 \%$ s at $24 \mathrm{~h}$ post-infection (Fig. 4C). Similar results were observed in the fluorescence intensity of AMC (Fig. 4D). The key apoptosis-associated proteins, including released CytC, cleaved caspase-3 and Bax, were examined using western blot analysis (Fig. 5A). Treatment with shRNA-NP-1 and shRNA-NP-2 inhibited the expression levels of released CytC, cleaved caspase- 3 and Bax. Following quantification of each band, it was found that shRNA-NP-1 and shRNA-NP-2

downregulated the expression of released $\mathrm{CytC}$ by $50 \%$ $(\mathrm{P}<0.05$; Fig. 5B). shRNA-NP-1 and shRNA-NP-2 treatment also downregulated the expression of cleaved caspase- 3 by at least $40 \%$ ( $\mathrm{P}<0.05$; Fig. 5C). The expression of Bax was significantly decreased by $\sim 50 \%$ by shRNA-NP-1 and shRNA-NP-2 (Fig. 5D).

\section{Discussion}

In the present study, the apoptosis of cardiomyocytes infected with H1N1pdm2009 was investigated. It was found that H1N1pdm2009 viral infection promoted the apoptosis of the H1N1pdm2009-infected H9C2 cells. The NP-specific shRNA significantly inhibited the viral infection and the virus-induced apoptosis of the $\mathrm{H} 9 \mathrm{C} 2$ cells. The results of the present study indicated the key pathogenic role of NP in H1N1pdm2009-induced apoptosis of the cardiomyocytes, and NP-specific shRNA may be an effective agent to prevent influenza virus-induced myocarditis.

It has been reported that influenza viruses can induce apoptosis in various cell types $(18,19)$, and several viral proteins, including M1, NS1 and PB1-F2 have also been shown to induce or inhibit apoptosis in human cells $(20,21)$. A previous report showed that the IAV may utilize its NS1 protein to interact with cellular $\beta$-tubulin to induce apoptosis (22). To date, few studies have been performed to investigate the role of NP in influenza-induced apoptosis. In the present study, it was found that shRNA targeting NP efficiently disrupted the production of H1N1pdm2009 virus, and markedly reduced the apoptotic rates of the infected H9C2 cells. In addition, key apoptosis-associated molecules, including released CytC, cleaved caspase-3 and Bax, were downregulated. These results indicated that NP has a key pathogenic role in H1N1pdm2009-induced apoptosis of cardiomyocytes.

Caspase-3 has been reported to be essential for viral replication in MDCK cells (23), and another study reported that the caspase-mediated cleavage of influenza NP in apoptosis has an antiviral effect (24). The results obtained in the present study indicated that shRNA against NP decreased the viral titer and effectively downregulated the level of cleaved caspase-3, and consequently suppressed the apoptotic rates of the cells. These results are in accordance with the previous conclusions that apoptosis may exert its protective effects not only by the elimination of infected cells, but also by the NP cleavage-mediated suppression of viral multiplication (24).

Numerous studies have indicated that cytokines are crucial in the induction of apoptosis in the heart (25), and it type I interferons are considered to cause influenza-induced apoptosis via a caspase-8-dependent mechanism (26). However, the exact pathogenic roles of cytokines during H1N1-induced apoptosis remain to be fully elucidated. The present study did not examine the apoptosis-associated cytokines in H1N1pdm2009 virus-infected animals; further investigations are required in animal models.

In conclusion, the present study demonstrated the vital pathogenic role of NP in the H1N1pdm2009-induced apoptosis of cardiomyocytes; NP-specific shRNA may be used as a potential therapeutic strategy to inhibit the influenza virus-induced apoptosis in cardiomyocytes and prevent influenza virus-associated acute myocarditis. 
A

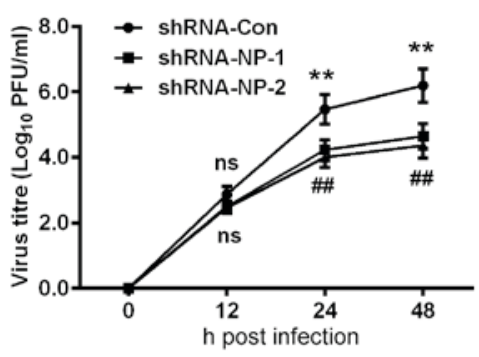

C

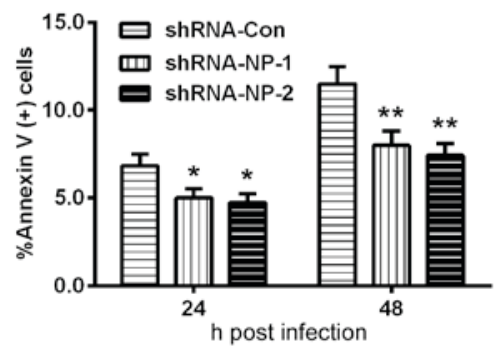

B

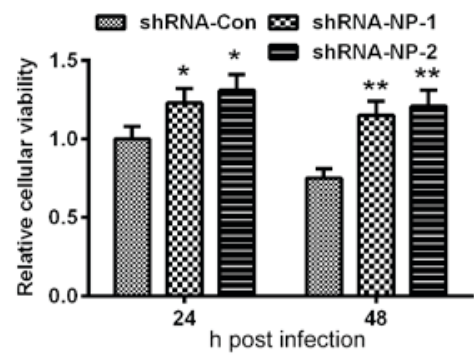

D

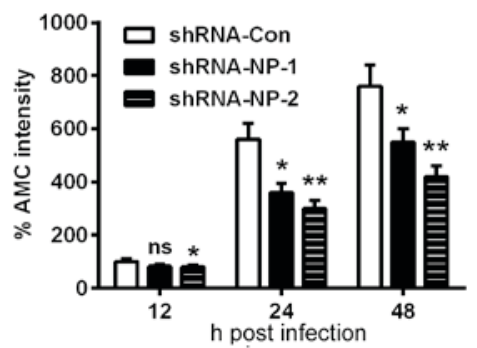

Figure 4. Apoptosis is induced by proliferation of the H1N1pdm2009 virus in H9C2 cells following NP knockdown. (A) Growth curve of H1N1pdm2009 virus in $\mathrm{H} 9 \mathrm{C} 2$ cells (MOI of 0.01 and 0.001 ), the viral titer at each time point was quantified using the plaque-forming assay following infection with 1 MOI Lenti-shRNA-NP-1, Lenti-shRNA-NP-2 or Lenti-shRNA-Con virus. Quantitative data are the average of three independent experiments. ${ }^{* *} \mathrm{P}<0.01$, Lenti-shRNA-NP-1 vs. Lenti-shRNA-Con; ${ }^{\# / P}$ < 0.01 , Lenti-shRNA-NP-2 vs. Lenti-shRNA-Con. Results of MTT assays for (B) cellular viability, (C) induction of apoptosis and (D) activity of caspase-3 in H9C2 cells infected with 1 MOI H1N1pdm2009 virus for 12, 24 or 48 h, following infection with 1 MOI Lenti-shRNA-NP-1, Lenti-shRNA-NP-2 or Lenti-shRNA-Con virus. Quantitative data are the average of three independent experiments. ${ }^{*} \mathrm{P}<0.05$, ${ }^{* *} \mathrm{P}<0.01$ and ${ }_{* * * *} \mathrm{P}<0.001$, as indicated. ns, no significance; NP, nucleoprotein; MOI, multiplicity of infection; shRNA, short hairpin RNA; con, control.

A

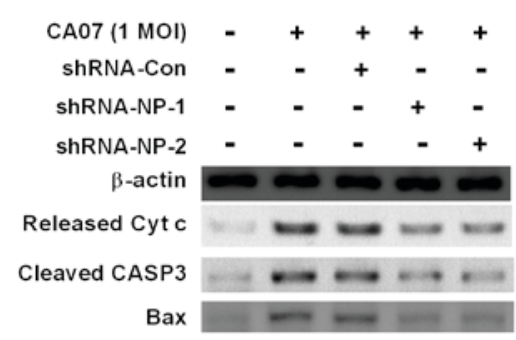

C

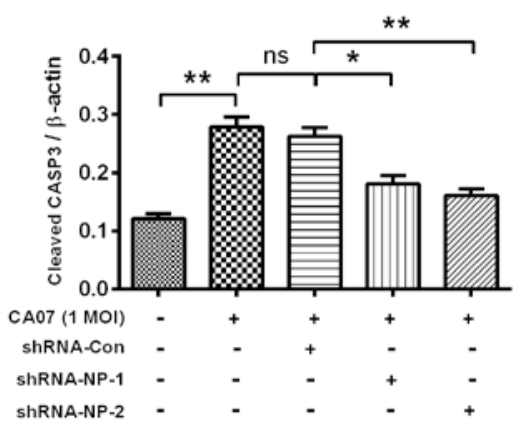

B

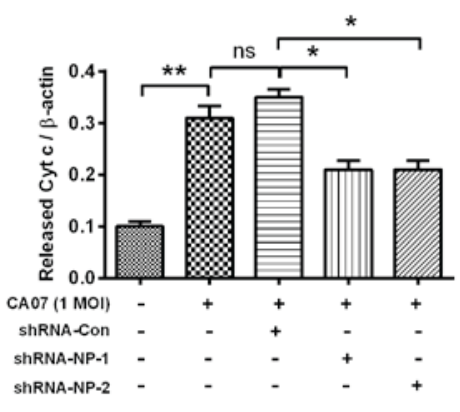

D

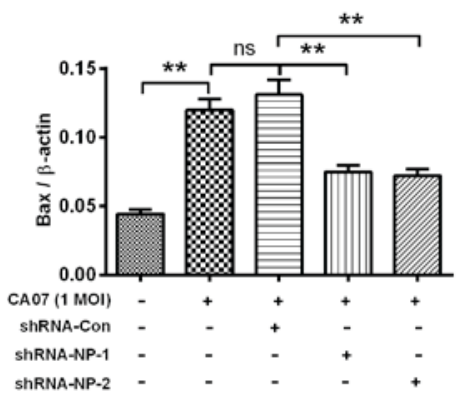

Figure 5. Western blot analyses of apoptosis-associated markers in H1N1pdm2009-infected H9C2 cells with or without NP-knockdown. (A) Representative western blot assay results of released Cytc from mitochondria, cleaved CASP3 or Bax in H1N1pdm2009-infected (1 MOI) H9C2 cells with or without NP-knockdown. Relative levels of (B) released Cytc, (C) cleaved CASP3 and (D) Bax inH1N1pdm2009-infected H9C2 cells with or without NP-knockdown. Quantitative data are the averaged of triplicate independent experiments. ${ }^{*} \mathrm{P}<0.05$ and ${ }^{* *} \mathrm{P}<0.01$. ns, no significance; NP, nucleoprotein; shRNA, short hairpin RNA; Cytc, cytochrome $c$, Bax, B cell lymphoma-2-associated X protein; MOI, multiplicity of infection; con, control. 


\section{References}

1. Fraser C, Donnelly CA, Cauchemez S, Hanage WP, Van Kerkhove MD, Hollingsworth TD, Griffin J, Baggaley RF, Jenkins HE, Lyons EJ, et al: Pandemic potential of a strain of influenza A (H1N1): Early findings. Science 324: 1557-1561, 2009.

2. Pan HY, Yano M and Kido H: Effects of inhibitors of Toll-like receptors, protease-activated receptor- 2 signalings and trypsin on influenza A virus replication and upregulation of cellular factors in cardiomyocytes. J Med Invest 58: 19-28, 2011.

3. Mamas MA, Fraser D and Neyses L: Cardiovascular manifestations associated with influenza virus infection. Int J Cardiol 130: 304-309, 2008

4. Warren-Gash C, Smeeth L and Hayward AC: Influenza as a trigger for acute myocardial infarction or death from cardiovascular disease: A systematic review. Lancet Infect Dis 9: 601-610, 2009.

5. Davis MM, Taubert K, Benin AL, Brown DW, Mensah GA, Baddour LM, Dunbar S and Krumholz HM; American Heart Association; American College of Cardiology: Influenza vaccination as secondary prevention for cardiovascular disease: A science advisory from the American Heart Association/American College of Cardiology. Circulation 114: 1549-1553, 2006.

6. Steinhauer DA, Domingo E and Holland JJ: Lack of evidence for proofreading mechanisms associated with an RNA virus polymerase. Gene 122: 281-288, 1992.

7. Ludwig S, Planz O, Pleschka S and Wolff T: Influenza-virusinduced signaling cascades: Targets for antiviral therapy? Trends Mol Med 9: 46-52, 2003

8. Ludwig S, Pleschka S, Planz O and Wolff T: Ringing the alarm bells: Signalling and apoptosis in influenza virus infected cells. Cell Microbiol 8: 375-386, 2006

9. Zhirnov OP, Konakova TE, Wolff T and Klenk HD: NS1 protein of influenza A virus down-regulates apoptosis. J Virol 76 $1617-1625,2002$

10. Mohsin MA, Morris SJ, Smith H and Sweet C: Correlation between levels of apoptosis, levels of infection and haemagglutinin receptor binding interaction of various subtypes of influenza virus: Does the viral neuraminidase have a role in these associations. Virus Res 85: 123-131, 2002.

11. Schultz-Cherry S and Hinshaw VS: Influenza virus neuraminidase activates latent transforming growth factor beta. J Virol 70 8624-8629, 1996.

12. Morris SJ, Nightingale K, Smith H and Sweet C: Influenza A virus-induced apoptosis is a multifactorial process: Exploiting reverse genetics to elucidate the role of influenza A virus proteins in virus-induced apoptosis. Virology 335: 198-211, 2005.
13. Zhirnov O and Bukrinskaya AG: Nucleoproteins of animal influenza viruses, in contrast to those of human strains, are not cleaved in infected cells. J Gen Virol 65: 1127-1134, 1984.

14. Zhirnov OP and Bukrinskaya AG: Two forms of influenza virus nucleoprotein in infected cells and virions. Virology 109: 174-179, 1981.

15. Ludwig S, Pleschka S and Wolff T: A fatal relationship-influenza virus interactions with the host cell. Viral Immunol 12: 175-196, 1999.

16. Pleschka S, Wolff T, Ehrhardt C, Hobom G, Planz O, Rapp UR and Ludwig S: Influenza virus propagation is impaired by inhibition of the Raf/MEK/ERK signalling cascade. Nat Cell Biol 3: 301-305, 2001

17. Livak KJ and Schmittgen TD: Analysis of relative gene expression data using real-time quantitative PCR and the 2(-Delta Delta C(T)) Method. Methods 25: 402-408, 2001.

18. Mori I, Komatsu T, Takeuchi K, Nakakuki K, Sudo M and Kimura Y: In vivo induction of apoptosis by influenza virus. J Gen Virol 76: 2869-2873, 1995

19. Roulston A, Marcellus RC and Branton PE: Viruses and apoptosis. Annu Rev Microbiol 53: 577-628, 1999.

20. Chanturiya AN, Basañez G, Schubert U, Henklein P, Yewdell JW and Zimmerberg J: PB1-F2, an influenza A virus-encoded proapoptotic mitochondrial protein, creates variably sized pores in planar lipid membranes. J Virol 78: 6304-6312, 2004.

21. Stasakova J, Ferko B, Kittel C, Sereinig S, Romanova J, Katinger H and Egorov A: Influenza A mutant viruses with altered NS1 protein function provoke caspase-1 activation in primary human macrophages, resulting in fast apoptosis and release of high levels of interleukins 1beta and 18. J Gen Virol 86: 185-195, 2005

22. Han X, Li Z, Chen H, Wang H, Mei L, Wu S, Zhang T, Liu B and Lin X: Influenza virus A/Beijing/501/2009(H1N1) NS1 interacts with $\beta$-tubulin and induces disruption of the microtubule network and apoptosis on A549 cells. PloS One 7: e48340, 2012.

23. Wurzer WJ, Planz O, Ehrhardt C, Giner M, Silberzahn T, Pleschka $\mathrm{S}$ and Ludwig S: Caspase 3 activation is essential for efficient influenza virus propagation. EMBO J 22: 2717-2728, 2003.

24. Zhirnov OP, Konakova TE, Garten W and Klenk H: Caspase-dependent $\mathrm{N}$-terminal cleavage of influenza virus nucleocapsid protein in infected cells. J Virol 73: 10158-10163, 1999.

25. Yajima T and Knowlton KU: Viral myocarditis: From the perspective of the virus. Circulation 119: 2615-2624, 2009.

26. Balachandran S, Roberts PC, Kipperman T, Bhalla KN, Compans RW, Archer DR and Barber GN: Alpha/beta interferons potentiate virus-induced apoptosis through activation of the FADD/Caspase-8 death signaling pathway. J Virol 74: $1513-1523,2000$ 